\title{
Acquired hemophilia and tuberculosis: case report and literature review
}

\begin{abstract}
Acquired hemophilia is a low-prevalence disease that is difficult to diagnose. It is of great importance to the health system because of the severity of and high costs related to its complications. It is characterized by the occurrence of spontaneous or traumatic hemorrhagic manifestations in different sites of the body, which are considered vital or potentially disabling emergencies due to the damages that they cause in various organs and the associated high mortality. We present the case of a male patient who developed acquired hemophilia $\mathrm{A}$, associated with an infectious disease. The patient was managed until he achieved total reduction of the inhibitor titers at the Medicarte IPS (Instituciones Prestadoras de Servicios de Salud, in Spanish [Health Providing Institutions]), Medellin, Colombia.
\end{abstract}

Keywords: hemophilia, hemorrhage, tuberculosis, infection, blood coagulation disorders, diagnosis, $\mathrm{AH}$
Volume 5 Issue 5 - 2017

\author{
Alejandro Uribe Toro,' Juan Guillermo \\ Duque Ortega ${ }^{2}$ \\ 'Medicarte IPS, Colombia \\ ${ }^{2}$ Department of Hematology, Medicarte IPS, Colombia
}

Correspondence: Juan Guillermo Duque Ortega, Hematologist Medicarte IPS, Carrera 43A \# 34-95 Almacentro Torre Sur Oficina 904, Medellín-Colombia, Fax +(4) 4484250 Tel +57 44842 50, Email mdgduque@hotmail.com

Received:September 20, 2017 | Published: December 27 2017
Abbreviations: PTT, partial thromboplastin time; AHA, acquired hemophilia A; CT, computed tomography; PCR, polymerase chain reaction; $\mathrm{BU}$, bethesda units; ICU, intensive care unit; RVVT, russell's viper venom time; SLE, systemic lupus erythematosus; PT, prothrombin time

\section{Introduction}

Acquired hemophilia ( $\mathrm{AH})$ is a rare disease with an estimated prevalence of 1.48 cases/million people/year and a mortality rate of $9-22 \%$, according to data obtained in the United Kingdom in a hemophilia surveillance study. ${ }^{1}$ Unlike congenital hemophilia, in which the presentation is exclusive to men, $\mathrm{AH}$ has no gender bias. It is characterized by the occurrence of spontaneous bleeding in different sites of the body, with rare hemarthrosis and muscle bleeding. Family history has not yet been associated with the development of the disease. $^{2}$

The diagnosis of $\mathrm{AH}$ is suspected in patients presenting spontaneous bleeding or bleeding with minimal trauma, with no family or personal history of coagulopathy. In the diagnostic algorithm, a PTT (Partial Thromboplastin Time) mixing test must be performed; a weak correction or no correction indicates AH. Then, the Russell's viper venom time (RVVT) is requested; a negative result indicates AH. From there, the level of inhibitor against factors VIII or IX is determined, confirming the diagnosis of AH. These results are accompanied by a decrease in the levels of coagulation factor VIII or IX. For the particular case, factor VIII deficiency is designated as acquired hemophilia A (AHA), ${ }^{2,3}$

The clinical features and manifestations of the alloantibodies known as inhibitors in congenital hemophilia differ from the autoantibodies or inhibitors of $\mathrm{AH}^{3,4}$ Soft tissue bleeding predominates in $\mathrm{AH}$ and is associated with the presence of other conditions or diseases. ${ }^{3,4}$ Autoimmune diseases such as rheumatoid arthritis, systemic lupus erythematosus (SLE), and thyroid disease have been associated with $\mathrm{AH}$. In turn, an association has been established with factors such as postpartum, neoplasias, infectious diseases, and some drugs. ${ }^{5,6}$
Acute bleeding in patients with AH is considered an emergency because of the high associated mortality. In different studies, between 8 and $22 \%$ of patients have died from bleeding. ${ }^{1,7-9}$ The management of bleeding events includes bridge therapy as the first line of treatment. The use of bypass agents such as recombinant activated factor VII and activated prothrombin complex derived from plasma are recommended. ${ }^{5,6,10}$ Other recommendations include avoiding invasive procedures, including combination therapies, such as the use of immunoglobulins and tranexamic acid, although the results are mixed. ${ }^{5,6}$

\section{Case presentation}

This case was a 67-year-old male patient with no family or personal history of coagulopathies, with a clinical presentation of right side pain associated with dyspnea and occasional fever since December 2014. A contrast chest computed tomography (CT) was performed that reported right pleural effusion accompanied by multiple nodular lesions in the right lung field and a mass in the apical region with dimensions of $5 \times 9.4 \times 8 \mathrm{~cm}$. A pleural biopsy was performed without hemorrhagic complications, with a report of chronic caseating granulomatous inflammation. The polymerase chain reaction (PCR) report was negative for tuberculosis, but there was a high suspicion of carcinomatosis.

After 4 months, the patient began to show clinical manifestations of ecchymosis in the neck and lower limbs. PT (prothrombin time) was reported as normal, and PTT (partial thromboplastin time) was prolonged at triple the level of the control. The diagnosis of coagulopathy was established, and AH was suspected, which was then confirmed by obtaining the level of inhibitors at 16BU (Bethesda Units). Specific management was not initiated at the beginning. A slow evolution was observed. Studies were again initiated that documented changes suggestive of chronic hepatitis without signs of cirrhosis or cervical and mediastinal lymphadenopathies accompanied by persistent pleural effusion. A cervical lymph node biopsy was performed, resulting in a report of chronic granulomatous inflammation with caseification necrosis and negative immunohistochemical 
markers for malignancy, suggestive of tuberculosis. Management with bypass agents was initiated prior to performing the lymph node biopsy, and 4 applications of FEIBA ${ }^{\circledR}$ were received before the biopsy. Postoperatively, hematoma was found in the neck with obstruction of the upper airway, which required orotracheal intubation and transfer to the intensive care unit (ICU). During the ICU stay, bypass therapy was initiated with NovoSeven ${ }^{\circledR}$ as follows: $4 \mathrm{mg}$ every 2 hours for 1 day, then $4 \mathrm{mg}$ every 3 hours for 2days; hemostasis control was achieved, followed by subsequent discharge and outpatient followup for tuberculosis management and no treatment for inhibitors, only watch and wait care.

In July 2015 , the patient was admitted to the program for patients with hemophilia at Medicarte. The medical management was monitored by a hematologist and the clinical laboratory, without pharmacological intervention. During the follow-up, there was a total reduction of the inhibitor titers and an increase of factor VIII levels up to a safe range (Table 1). No spontaneous bleeding occurred during follow-up.

Table I Clinical laboratory follow-up

\begin{tabular}{lll}
\hline Date & Factor VIII levels (\%) & $\begin{array}{l}\text { Factor VIII } \\
\text { inhibitors (BU) }\end{array}$ \\
\hline April 2I, 20I5 & 0.6 & 16.4 \\
July 16, 20I5 & 5 & No data \\
October 08, 20I5 & 15 & 0 \\
January 28, 2016 & 58 & 0 \\
\hline
\end{tabular}

Source: Clinical history

\section{Discussion}

At present, three cases of AHA-associated pulmonary or extra pulmonary tuberculosis have been documented. The established treatment method involved plasmapheresis or immunosuppression, with successful results. ${ }^{11-13}$ In the case presented, there was evidence of an improvement in factor VIII levels and the disappearance of inhibitor titers during the anti-tuberculosis treatment, without the need for additional measures. Bypass therapy was only required during the invasive procedures that were performed for the diagnosis of the underlying disease.

In our setting, there may be a higher number of cases of acquired hemophilia associated with infectious diseases, particularly tuberculosis, due to the high prevalence of TB, which was 33 cases per 100,000 inhabitants in $2014 .{ }^{14}$ However, it is not diagnosed more often because of difficulties in accessing medical care and because of the ignorance of the existence of such a pathology and its associations with other diseases by health personnel.

The treatment of AHA is based on two main objectives: the management of acute bleeding and the elimination of inhibitors. ${ }^{2,5}$ Several therapies for the elimination of inhibitor titers have been recognized so far, without a standard management guide being established. First-line treatment includes pharmacological management, in combination or alone, using immunosuppressant's with steroids, rituximab, cyclophosphamide, and calcineurin inhibitors; $5,6,15-18$ the use of immunoglobulins; ${ }^{19}$ and control of the underlying pathology. ${ }^{20}$ Even spontaneous remissions have been documented. ${ }^{21}$

The development of inhibitors is an uncommon condition.
Consequently, recommendations derived from studies have been limited. Thus, the management is based on the conclusions of a few studies and the opinions of experts. In the case presented, the resolution of AHA was the result of the control and management of the infectious disease. The use of bridge therapy with the bypass agents was limited only to the diagnostic procedure. The lack of microbiological isolation of tuberculosis is possibly explained by the association between tuberculosis and sarcoidosis because it is considered a representation of a spectrum of the same disease. ${ }^{22}$ After the initiation of the anti-tuberculosis treatment, an adequate clinical response was observed, with the disappearance of lymphadenopathies and resolution of the AHA.

\section{Conclusion}

When facing a case of $\mathrm{AH}$, it is necessary to initiate tests in search of underlying pathologies. The treatment should focus on the control and management of the associated pathology, without the need to resort to expensive and/or higher risk therapies, such as immunosuppressive therapies and the use of biological agents, which should be used in the management of the bleeding or in preparation for performing invasive procedures. More studies of this pathology are required because the recommendations are still unclear due to the low incidence and prevalence. The objective should be focused on providing information to the specialist that permits decision-making regarding the diagnosis and treatment.

\section{Acknowledgements}

To Medicarte IPS and EPS SURA for their commitment to generate knowledge.

\section{Conflict of interest}

The authors have no conflicts of interest to declare.

\section{Funding}

This work was supported by IPS Medicarte, Colombia.

\section{References}

1. Collins PW, Hirsch S, Baglin TP, et al. Acquired hemophilia A in the United Kingdom: a 2-year national surveillance study by the United Kingdom Haemophilia Centre Doctors' Organisation. Blood. 2007;109(5):1870-1877.

2. Craig M Kessler. Acquired inhibitors to factor VIII. In: Christine A Lee, et al. editors. Textbook of Hemophilia. Oxford, UK: Wiley Blackwell; 2014. p. 85-94.

3. Peter Collins, Francesco Baudo, Angela Huth-Kühne, et al. Consensus recommendations for the diagnosis and treatment of acquired hemophilia A. BMC Res Notes. 2010;3:161.

4. Srivastava A, Brewer AK, Mauser-Bunschoten EP, et al. Guidelines for the management of hemophilia. Haemophilia. 2013;19(1):e1-47.

5. Kessler CM, Knöbl P. Acquired haemophilia: an overview for clinical practice. Eur J Haematol. 2015;95 Suppl 81:36-44.

6. Collins PW, Chalmers E, Hart D, et al. Diagnosis and management of acquired coagulation inhibitors: a guideline from UKHCDO. $\mathrm{Br} J$ Haematol. 2013;162(6):758-773.

7. Huang SY, Tsay W, Lin SY, et al. A study of 65 patients with acquired hemophilia A in Taiwan. J Formos Med Assoc. 2015;114(4):321-327.

8. Franchini M, Lippi G. Acquired factor VIII inhibitors. Blood. 2008;112(2):250-255. 
9. Tiede A, Klamroth R, Scharf RE, et al. Prognostic factors for remission of and survival in acquired hemophilia A (AHA): results from the GTHAH 01/2010 study. Blood. 2015;125(7):1091-1097.

10. Zanon E, Milan M, Gamba G, et al. Activated prothrombin complex concentrate $(\operatorname{FEIBA}(\mathrm{R}))$ for the treatment and prevention of bleeding in patients with acquired haemophilia: a sequential study. Thromb Res. 2015;136(6):1299-1302.

11. Aurousseau MH, Eclache V, Fain O, et al. Acquired factor VIII inhibitor in a patient with tuberculosis. Haemostasis. 1996;26(6):319-320.

12. Ogata H, Sakai S, Koiwa F, et al. Plasma exchange for acquired hemophilia: a case report. Ther Apher. 1999;3(4):320-322.

13. Esteve Atienzar PJ, Salas de Zayas R, Ferrufino Rivera E, et al. Acute renal failure induced by acquired hemophiliac syndrome. Medicina Intensiva. 2011;35(9):586-587.

14. World health organization. Global Tuberculosis Report; Incidence of tuberculosis 2000-2015. USA: World Bank Group; 2016.

15. Collins P, Baudo F, Knoebl P, et al. Immunosuppression for acquired hemophilia A: results from the European Acquired Haemophilia Registry (EACH2). Blood. 2012;120(1):47-55.
16. D'arena G, Grandone E, Di Minno MN, et al. The anti-CD20 monoclonal antibody rituximab to treat acquired haemophilia A. Blood Transfus. 2016;14(2):255-261.

17. Franchini M, Mannucci PM. Inhibitor eradication with rituximab in haemophilia: where do we stand? Br J Haematol. 2014;165(5):600-608.

18. Pardos-Gea J, Altisent C, Parra R, et al. Acquired haemophilia A. First line treatment with calcineurin inhibitors and steroid pulses: a 10-year follow-up study. Haemophilia. 2012;18(5):789-793.

19. Schwartz RS, Gabriel DA, Aledort LM, et al. A prospective study of treatment of acquired (autoimmune) factor VIII inhibitors with high-dose intravenous gammaglobulin. Blood.1995;86(2):797-804.

20. Mariotti J, Locatelli G, Cirrincione S, et al. Eradication of acquired hemophilia associated with indolent non-Hodgkin lymphoma by a disease specific treatment. Leuk Lymphoma. 2015;56(11):3210-3212.

21. Lottenberg R, Kentro TB, Kitchens CS. Acquired hemophilia. A natural history study of 16 patients with factor VIII inhibitors receiving little or no therapy. Arch Intern Med. 1987;147(6):1077-1081.

22. Agrawal R, Kee AR, Ang L, et al. Tuberculosis or sarcoidosis: opposite ends of the same disease spectrum? Tuberculosis (Edinb). 2016;98:2126. 